\section{One Parameter Bifurcation Diagram for Chua's Circuit}

MIREILLE E. BROUCKE

\begin{abstract}
The objective of this letter is to report on the usefulness of a 1-dimensional map for characterizing the dynamics of a third-order piecewise-linear circuit. The map is used to reproduce period-doubling birfurcations and particularly to compute Feigenbaum's number. The 1-dimensional map gives qualitatively identical results as the circuit equations and proved to be far more computationally efficient.
\end{abstract}

A 1-dimensional map was applied to performing an approximate analysis of a third-order piecewise-linear chaotic circuit. Several positive results made this approach an appealing form of analysis: 1) the 1-dimensional map correctly predicted all qualitative behavior for the circuit, namely periodic windows, onset of the double scroll attractor, disappearance of the attractor, existence of a saddle-type periodic orbit, and conditions for a homoclinic orbit [1]; 2) it dramatically reduced the computation time of diagrams depicting dynamic bifurcations; and 3) it made possible an efficient and simple means to compute Feigenbaum's constant. With these reasons motivating a more in-depth discussion of the 1-dimensional map, the framework in which this map is formulated, some of its special properties, and results obtained by it will be presented. We will emphasize the map's interpretation as representing the behavior of a physical circuit.

The autonomous differential equations in dimensionless form for Chua's circuit are

$$
\begin{aligned}
& \dot{x}=\alpha(y-h(x)) \\
& \dot{y}=x-y+z \\
& \dot{z}=-\beta y
\end{aligned}
$$

where the piecewise-linear term is

$$
h(x):=m_{1} x+\frac{m_{0}-m_{1}}{2}(|x+1|-|x-1|) .
$$

In what follows, $m_{0}=-1 / 7$ and $m_{1}=2 / 7$, while $\alpha$ and $\beta$ are the bifurcation parameters.

The important feature of this equation is that the state space is divided into three linear (or affine) regions, each containing an equilibrium point. One can identify a complex eigenplane and real eigenvector corresponding to a pair of complex eigenvalues and a real eigenvalue, respectively, for each region of the vector field. The vector field under consideration is constrained so that the eigenspaces of either the real or complex eigenvalues are not parallel to the $x=-1$ or $x=1$ boundary planes. A possible geometry of the eigenspaces is depicted in Fig. 1.

The 1-dimensional map is based on observations of the special properties of the chaotic attractor (called the double scroll) in Chua's circuit. Hence, it is insightful to examine the dynamics of the circuit. A good understanding can be derived simply from a closer inspection of Fig. 1. Keep in mind that the eigenspaces are invariant sets, so trajectories cannot pass through them.

A restricted set of eigenvalues (or parameter values $-\alpha, \beta$, $\left.m_{0}, m_{1}\right)$ is allowed to ensure continuity of the vector field at the boundaries and to guarantee the existence of the double scroll

Manuscript received August 14, 1986; revised October 7, 1986.

The author is with the Department of Electrical Engineering and Computer Science, University of California, Berkeley, CA 94720.

IEEE Log Number 8611949

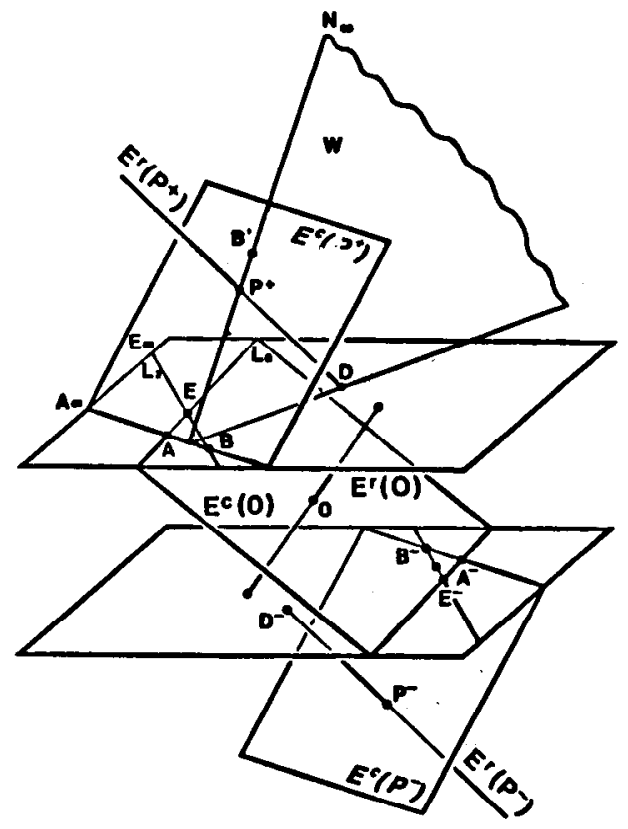

Fig. 1. Eigenspaces for the three regions of Chua's circuit.

attractor. For continuity, the real eigenvalues of adjacent regions. must have opposite stability properties. To obtain the double scroll, we must have the real eigenvalue in the middle region unstable and the complex eigenvalues in the top region unstable. Note that the vector field is symmetric so it suffices to discuss only the top and middle regions. It is easy to prove that the points where the vector field in the $x=1$ plane are parallel to that plane compose a line, labeled $L_{2}$ in Fig. 1. It also logically follows that this line represents points of tangency of trajectories to the $x=1$ plane. Moreover, enough information had been given to convince oneself that all trajectories to the right of $L_{2}$ pass the $x=1$ plane in an increasing- $x$ direction, while trajectories to the left of $L_{2}$ pass "down" through the boundary plane.

The following three facts are now apparent. 1) Trajectories originating in the triangle $A B E$ pass to the middle region and reenter the top region without entering the lower region; trajectories passing through the wedge $\square A_{\infty} A E E_{\infty}$ will continue to the bottom region. 2) Since the double scroll has been observed to span all three regions, it must have trajectories to the left and to the right of line $L_{0}$. 3) Trajectories emanating from $\square A_{\infty} B E_{\infty}$ will eventually return to it; hence, it forms a natural boundary for defining a Poincaré map.

With one more piece of information, the setting will be complete for defining the 1-dimensional map: for the parameter values of interest (for which the double scroll attractor has been observed), the real stable eigenvalue in the upper region is relatively large in absolute value compared to the other eigenvalues. So trajectories are rapidly contracted to the complex eigenplane of $P+$ and will transfer to the middle region very close to the line $\overline{B A}$.

The 1-dimensional map is defined as

$$
\tilde{\pi}^{*}: \overrightarrow{B A_{\infty}} \rightarrow \overrightarrow{B A_{\infty}} \text {. }
$$

To ensure that the 1-dimensional map is continuous and maps to unique points, we require that trajectories emanating from $\square A_{\infty} A E E_{\infty}$, upon returning to the top region, do not hit the line $L_{2}$ (i.e., the trajectory will transfer to the top region, as opposed 


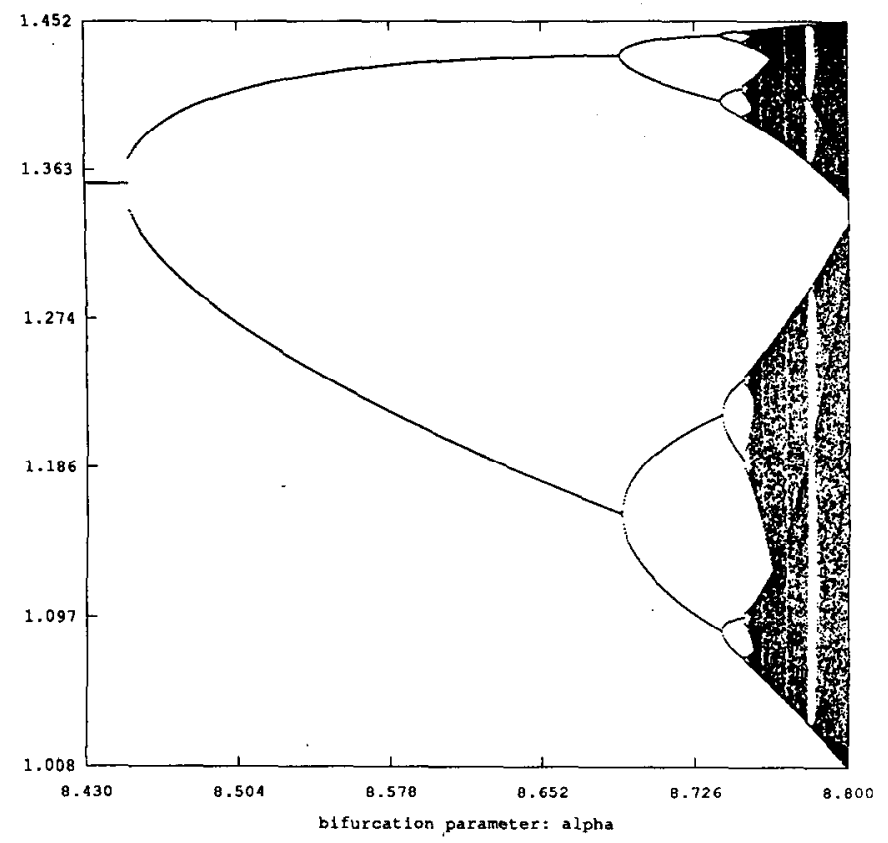

Fig. 2. Bifurcation diagram using the 1-dimensional map, showing the period-doubling route to chaos.

to being tangent to $x=1$ and remaining in the middle region). Secondly, we assume that trajectories emanating from $\square A_{\infty} A E E_{\infty}$ return to the $x=1$ plane to the right of point $D$ in Fig. 1 . Considering the plane $W$, the second assumption guarantees that trajectories will pass through $W$. Moreover, they are compressed closely to the ray $\overrightarrow{P+N_{\infty}}$. Hencc, we can define an equivalent but simpler 1-dimensional map

$$
\pi^{*}: \overrightarrow{P+N_{\infty}} \rightarrow \overrightarrow{P+N_{\infty}} \text {. }
$$

This definition is preferred since it yields a simpler analytic expression [1].

We have justified the use of the 1-dimensional map for its applicability to studying the double scroll attractor, but it deserves mention that this map has independent interest to those investigating properties of 1-dimensional maps. For example, there is a unique point $B^{\prime}$ on $\overrightarrow{P+N_{\infty}}$ that maps to $B$; initial conditions between $P+$ and $B^{\prime}$ do not intersect $\overrightarrow{B A} A_{\infty}$ but map to a point on $\overrightarrow{P+N_{\infty}}$ so that the segment of $\pi^{*}$ defined on $\overrightarrow{P+B^{\prime}}$ is linear. The analytic expressions for points on $\overrightarrow{B^{\prime} N_{\infty}}$ are more involved and are based on Poincaré maps. Poincaré half maps are defined on $\square A_{\infty} B E_{\infty}$ mapping points in that region to their first return points on the $x=1$ boundary plane. The reader is referred to [1] for a more complete description.

Fig. 2 (see [1, fig. 29(b)] for a multicolor version) demonstrates an application of the 1-dimensional map for portraying dynamic bifurcations. The final objective of this letter is to present this figure and report how it was generated.

The diagram, illustrating the period-doubling route to chaos in Chua's circuit, is produced by fixing $\beta=15$ and varying $\alpha$ from 8.43 to 8.8 . For each $\alpha$ value, the 1-dimensional map is computed and a table is formed of $x, \pi^{*}(x)$ values. The program iterates the map (interpolating between data points in the table) until transient behavior is eliminated. A fixed number of points are thereupon plotted, and the process is repeated for the next $\alpha$ value. The map greatly reduces the computation time to generate a bifurcation diagram. When compared to a "brute force" diagram, obtained by integrating (1), one observes qualitatively similar behavior, but the 1-dimensional map diagram has better resolution, whereas the circuit equations produce a "fuzzy" diagram. This fuzziness, despite a large number of transient iterations, is a result of the relative crror used in the integration method. The variables plotted on the $y$-axis of the two diagrams are related by a coordinate transformation. Two photos of a 1-dimensional map diagram and a brute force diagram depicting these differences are included in [1].

The clarity of the 1-dimensional map diagram facilitated finding the bifurcation points to compute Feigenbaum's constant, i.e., the inverse rate of period-doubling bifurcations. The method goes as follows. The program determines the period (1-32) for each $\alpha$ value. When the period double occurs, the program steps back and does a more precise check for the parameter value where the period changed by reducing the $\alpha$-stepsize. The bifurcation points are used after the simulation is complete to compute the rate. The most accurate measurement yielded 4.6933.

\section{REFERENCES}

[1] L. O. Chua, M. Komurn, and T. Matsumoto, "The double scroll family," IEEE Trans. Circuits 'yst., vol. CAS-31, pp. 1072-1118, Nov. 1986.

\section{Another Proof of a Property of Multivariable Bilinear Transformation Matrices}

\section{DAVID HERTZ AND EZRA ZEHEB}

Abstract-A new proof is provided to a general property of multivariable bilinear transformation matrices. The proof becomes immediate by using a formal rule for operating with Kronecker products.

The multivariable bilinear transformation

$$
z_{i}=\frac{s_{i}+1}{s_{i}-1} \quad s_{i}=\frac{z_{i}+1}{z_{i}-1}, \quad i=1, \cdots, n
$$

of a multivariable polynomial

$$
A(z)=A\left(z_{1}, \cdots, z_{n}\right)
$$

is useful in various engineering applications, e.g., design and stability tests of multidimensional digital filters. The transformed polynomial $B(s)$ is defined by

$$
B(s)=B\left(s_{1}, \cdots, s_{n}\right)=\prod_{i=1}^{n}\left(s_{i}-1\right)^{N_{i}} A\left(\frac{s_{1}+1}{s_{1}-1}, \cdots, \frac{s_{n}+1}{s_{n}-1}\right)
$$

Manuscript received February 26, 1986; revised June 30, 1986. The research of E. Zeheb was supported in part by the Air Force Wright Aeronautical Laboratories, United States Air Force, under Grant AFOSR-84-0344.

D. Hertz is with the Department of Electrical Engineering, Technion-Israel Institute of Technology, Haifa, Israel.

E. Zeheb is with the Faculty of Engineering, Tel-Aviv University, Tel-Aviv, Israel, on leave of absence from the Technion-Israel Institute of Technology Haifa, Israel.

IEEE Log Number 8610870 . 